\title{
Optical properties of carbon nanostructures produced by laser irradiation on chemically modified multi-walled carbon nanotubes
}

Article in Optics \& Laser Technology • October 2016

DOI: $10.1016 /$ j.optlastec.2016.05.002

CITATIONS

3

8 authors, including:
READS

98

\section{Susana Hernández-López}

Universidad Autónoma del Estado de México. 31 PUBLICATIONS 266 CITATIONS

SEE PROFILE

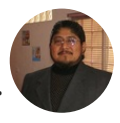

Rurik Farias

Universidad Autónoma de Ciudad Juárez

46 PUBLICATIONS 42 CITATIONS

SEE PROFILE

\section{Claudia Alejandra Hernández-Escobar}

Centro de Investigación en Materiales Avanza...

22 PUBLICATIONS 213 CITATIONS

SEE PROFILE

\section{E. Armando Zaragoza-Contreras}

Centro de Investigación en Materiales Avanz... 37 PUBLICATIONS 380 CITATIONS

SEE PROFILE

Some of the authors of this publication are also working on these related projects:

Nanophotonics View project 
Full length article

\title{
Optical properties of carbon nanostructures produced by laser irradiation on chemically modified multi-walled carbon nanotubes
}

\author{
Enrique Vigueras Santiago a , Susana Hernández López a , Marco A. Camacho López a \\ Delfino Reyes Contreras ${ }^{\mathrm{b}}$, Rurik Farías-Mancilla ${ }^{\mathrm{c}}$, Sergio G. Flores-Gallardo ${ }^{\mathrm{d}}$, \\ Claudia A. Hernández-Escobar ${ }^{\mathrm{d}}$, E. Armando Zaragoza-Contreras ${ }^{\mathrm{d}, *}$ \\ a Laboratorio de Investigación y Desarrollo de Materiales Avanzados, Facultad de Química, Universidad Autónoma del Estado de México, Campus Rosedal, \\ Km 14.5 Carretera Toluca Atlacomulco, San Cayetano de Morelos, Toluca C.P. 50925, Mexico \\ ${ }^{\mathrm{b}}$ Department of Physics and Electrical Engineering, University of North Texas, Denton, TX, USA \\ c Laboratorio de Investigación y Desarrollo de Materiales Avanzados (LIDMA), Facultad de Química, Universidad Autónoma del Estado de México, \\ Paseo Colón esquina Paseo Tollocan, Toluca Estado de México CP50000, México \\ d Department of Chemical Engineering and Materials, Centro de Investigación en Materiales Avanzados, S.C. Chihuahua, Chih., México
}

\section{A R T I C L E I N F O}

\section{Article history:}

Received 2 November 2015

Received in revised form

15 March 2016

Accepted 6 May 2016

\section{Keywords:}

Carbon nanostructures

Carbon nanotubes

Laser irradiation

Optical properties

Photoluminescence

\begin{abstract}
A B S T R A C T
This research focused on the nanosecond (Nd: YAG-1064 $\mathrm{nm}$ ) laser pulse effect on the optical and morphological properties of chemically modified multi-walled carbon nanotubes (MWCNT). Two suspensions of MWCNT in tetrahydrofuran (THF) were prepared, one was submitted to laser pulses for 10 min while the other (blank) was only mechanically homogenized during the same time. Following the laser irradiation, the suspension acquired a yellow-amber color, in contrast to the black translucent appearance of the blank. UV-vis spectroscopy confirmed this observation, showing the blank a higher absorption. Additionally, photoluminescence measurements exhibited a broad blue-green emission band both in the blank and irradiated suspension when excited at $369 \mathrm{~nm}$, showing the blank a lower intensity. However, a modification in the excitation wavelength produced a violet to green tuning in the irradiated suspension, which did not occur in the blank. Lastly, the electron microscopy analysis of the treated nanotubes showed the abundant formation of amorphous carbon, nanocages, and nanotube unzipping, exhibiting the intense surface modification produced by the laser pulse. Nanotube surface modification and the coexistence with the new carbon nanostructures were considered as the conductive conditions for optical properties modification.
\end{abstract}

c) 2016 Elsevier Ltd. All rights reserved.

\section{Introduction}

Nanostructured materials display unexpected and unusual properties not observed in the macroscopic scale; for example, plasmon resonance [1], tunable light emission [2], or biocompatibility [3]. Generally, nanomaterials properties can be controlled by the synthesis route. Consequently, several chemical, biological, and physical methods of synthesis have been successfully implemented; however, nowadays the demand of nanomaterials for diverse applications is still an important research challenge [4]. Nanomaterials production through physical techniques, primarily those assisted by laser pulses, has gained great attention in the last decades due to its purity (no need for additives) and speed, and because nanomaterials from almost any source are produced [5-8].

\footnotetext{
* Corresponding author.

E-mail address: armando.zaragoza@cimav.edu.mx (E.A. Zaragoza-Contreras).
}

One of these routes involves laser pulse incidence on solid targets confined in a liquid. Here, the pulse produce ablation, that is, material detachment (ions, clusters, etc.) from which the nanomaterials are formed [5,7]. This technique, referred as pulsed laser ablation in a liquid phase, has demonstrated its utility for long to produce a variety of nanomaterials [8-10].

An alternative way to produce nanomaterials, using laser pulses, consists of preparing a suspension of the desired source in a solvent and submit it to laser pulses; laser pulses induce reshaping, resizing or fragmentation of the suspended material. As additives are not required, this method presents several advantages as high purity nanoparticles are obtained. Under this concept, nanoparticle suspension in a liquid, as target material, presents the advantage of the coexistence of the nanomaterial produced by the laser pulses and the rests of the original, with the consequent modification of, for instance, luminescence of the entire solution [9]. Through the laser fragmentation in suspension (LFS) 
technique, the size reduction of gold nanoparticles [11], fragmentation of indium tin oxide nanoparticles into smaller ones [12], the evolution of $\mathrm{ZnO}$ hollow nanospheres into $\mathrm{ZnO}$ quantum dots [13] and others, was reported. Detailed description of the evolution of ablation mechanisms of solid and particle targets confined in liquid phase are reported in literature [10, 14-16].

Concerning carbon nanomaterials (CNM), LFS methodology has been employed during the last two decades to produce a variety of carbon structures, for instance, polyynes [17-19], carbon nanoparticles $[9,20-22]$, carbon nanospindles $[10,23]$, carbon nanocages [9], carbon nanodiamonds [20,24,25], or nanocubes [26]. For this purpose, diverse sources of carbon as, for instance, $C_{60}$ [17], diamond particles [19], graphite powders [20], or carbon black $[9,27]$, were confined either in organic solvents or in water and then laser irradiated. In these studies, the driving forces which define the nanoparticle morphology were mostly related to laser power, incidence time, solvent, and carbon source morphology. The production of CNM represents an emergent option for hightech application as compared with quantum dots, photoluminescent CNM are more chemically stable, inert, and biocompatible [28-32]. Accordingly, research aimed at the development of new strategies to obtain new forms of CNM with active optical properties is of great interest.

For the best of our knowledge, the only report on the use of LFS technique for the modification of CNT in suspension deals with the bleaching of an SWCNT/DMF solution after its irradiation with ns laser pulse [33]. Herein, we report on the laser pulse effect using as the target chemically modified multi-walled carbon nanotubes (MWCNT) suspended in tetrahydrofuran (THF). The purpose of using modified nanotubes was to improve the dispersibility in the liquid phase, besides of providing products of certain functionalization. The possibility to produce carbon nanostructures such as polyynes, carbon nanoparticles, nanodiamonds, or carbon nanocages using LFS technique from MWCNT is discussed. The morphology and optical activity of the ablation products were characterized by electron microscopy, UV-vis and photoluminescence (PL) measurements.

\section{Experimental}

\subsection{Materials and laser irradiation}

In this work, MWCNT (Aldrich Co.) were oxidized in acid medium following the method reported previously [34]. THF (Aldrich Co.) was used as received. For laser irradiation, $5 \mathrm{mg}$ of MWCNT was dispersed in $20 \mathrm{~mL}$ of THF; the dispersion was irradiated inside a conical vial for $30 \mathrm{~min}$, using a $1064 \mathrm{~nm}$ laser NdYAG (Minilite II, Continuum), pulse duration was $7 \pm 2$ ns at $15 \mathrm{~Hz}$ of repetition frequency, pulse energy was $50 \mathrm{~mJ} /$ pulse. A blank suspension of MWCNT dispersed mechanically in THF was prepared for comparison purposes. Fig. 1 shows the experimental setup. During the laser irradiation, neither control of pressure or temperature was intended.

\subsection{Measurements}

Optical absorption spectra of the MWCNT suspensions, after and before laser irradiation, were run using a double beam spectrometer (Lambda7, Perkin-Elmer) from 200 to $900 \mathrm{~nm}$. Absorbance spectra were recorded in a quartz cuvette with an optical path length of $10 \mathrm{~mm}$. For reference purposes, THF absorption spectrum was recorded. All spectra were compared with the air. Photoluminescence (PL) characterization was carried out using a spectrophotometer (FluoroMax Plus, Horiba) exciting the MWCNT suspension at various wavelengths. The PL spectrum of the quartz



Fig. 1. Experimental setup for the laser fragmentation.

cell filled with pure THF was also obtained. All the experiments were performed at environmental conditions without any special monitoring or control. MWCNT morphology, before and after irradiation, was analyzed using a field emission scanning electron microscope (FE-SEM, JSM-7401 F, JEOL Ltd.) and a field emission transmission electron microscope (JEM 2200FS, JEOL Ltd.). The samples were prepared by placing a droplet of dispersion on a holey-carbon-copper grid and then evaporating the solvent under laboratory conditions.

\section{Results and discussion}

\subsection{Optical properties}

During laser incidence, nanotube suspension evidenced changes in its optical properties, as the initial translucent black suspension turned progressively into a pale amber color (inset in Fig. 2). UV-vis absorbance and PL of the irradiated suspension were firstly analyzed. Fig. 2 exhibits MWCNT absorbance spectra, after and before irradiation, and pure THF. As seen, THF is nearly transparent from 340 to $850 \mathrm{~nm}$; however, after MWCNT addition turned into a translucent black suspension $\left({ }^{*}\right)$, as portrayed in the inset picture. Concerning the irradiated dispersion, a great difference is seen compared to the blank. Absorbance decreased considerably from 450 to $850 \mathrm{~nm}$, indicating that the dispersion evolved to a lower turbidity, as corroborated from the inset picture where the dispersion shows a pale-amber color $(+)$. Such a change was related to the modification of MWCNT nanostructure, or to the coexistence of a new form of carbon nanostructure with the MWCNT. Absorbance modifications have been correlated to the evolution of products derived from the original nanomaterial irradiated. Mikheev et al. reported that the optical properties of MWCNT suspended in DMF change significantly after laser irradiation; the suspension was bleached and its optical density 


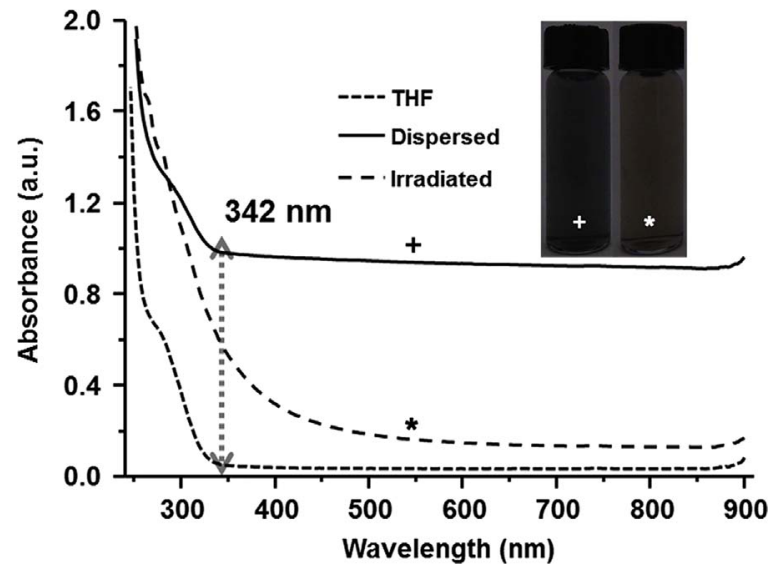

Fig. 2. Absorbance spectra of MWCNT suspensions before $\left(^{*}\right)$ and after $(+)$ irradiation. THF spectrum is also included as a reference. The inset illustrates dispersions appearance under white light.

reduced in a wide wavelength range from 225 to $900 \mathrm{~nm}$. They showed that bleaching was due to laser stimulated chemical reactions between DMF molecules and MWCNT [33]. Concerning other nanomaterials, Usui et al. reported that fragmented $\mathrm{TiO}$ nanoparticles showed higher transmittance in the visible region compared with the non-fragmented ones [12]. Tarasenko et al. [35] reported plasmon maximum shifts to longer wavelengths with a decrease of the peak height in gold ( $\mathrm{Au}$ ) nanoparticles, produced by laser ablation of solid target in aqueous phase. Spectral changes were attributed to Au nanoparticle progressive agglomeration after laser irradiation was switched off.

Literature indicates that in CNM, such as nanodiamonds or carbon dots, photoluminescence is highly dependent on particle size [22,36,37]. Accordingly, to corroborate PL emission, a study on MWCNT suspensions before and after laser pulses was conducted, Fig. 3. Emission spectra showed, respectively, broad bands ranging from $380 \mathrm{~nm}$ to $525 \mathrm{~nm}$ and from $380 \mathrm{~nm}$ to $650 \mathrm{~nm}$ for the blank and irradiated suspension. A blue-green color of the irradiated solution can be appreciated from the inset in Fig. 3. Both spectra are characterized by two peaks located at $410 \mathrm{~nm}$ and $432 \mathrm{~nm}$, and THF does not show PL emission. Fig. 4 exhibits excitation wavelength effect on PL of irradiated MWCNT suspension; emission band shifting to the red color (region), when the excitation wavelength increased, suggesting the possibility to tune the PL

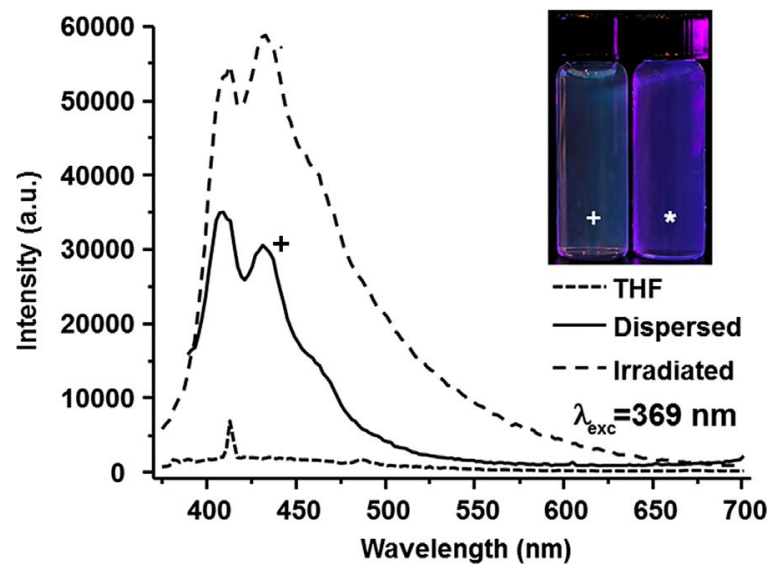

Fig. 3. PL intensity of the MWCNT dispersion under $369 \mathrm{~nm}$ excitation wavelength, $\left({ }^{*}\right)$ before and after $(+)$ irradiation. The inset shows the suspension under $369 \mathrm{~nm}$ excitation. (For interpretation of the references to color in this figure, the reader is referred to the web version of this article.)

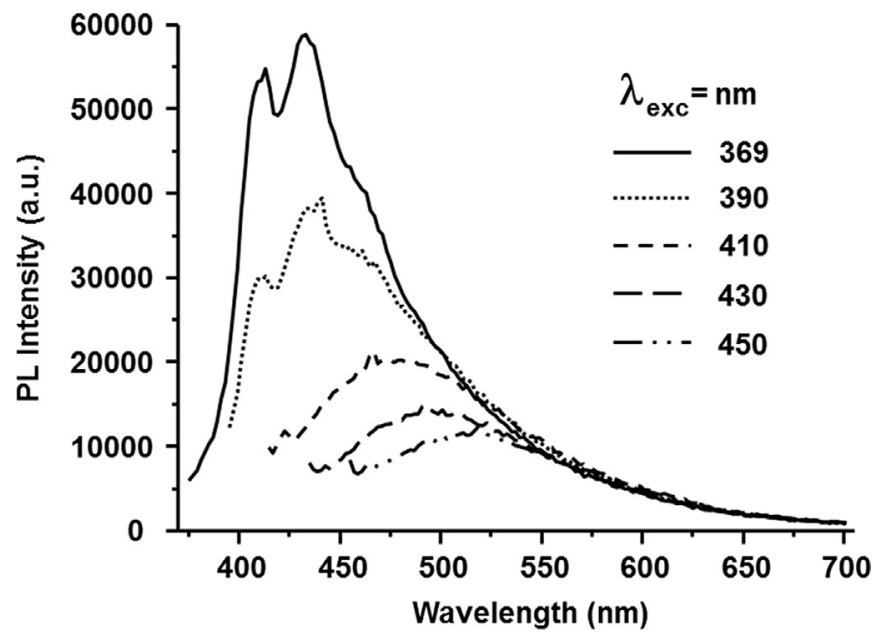

Fig. 4. PL emissions of irradiated MWCNT under different excitation wavelength (For interpretation of the references to color in this figure, the reader is referred to the web version of this article.)

emission. The same experiment achieved on the blank exhibited no effect. Furthermore, the peaks at $410 \mathrm{~nm}$ and $432 \mathrm{~nm}$ disappeared at a larger excitation wavelength, showing also a change in the spectrum shape. Again, we related this difference to the generation of new carbon species, for example, carbon nanoparticles, or by the modification of the original nanotubes (nanotube fragmentation). As mentioned, PL is highly affected by particle size reduction, Hu et al. [9,27] reported the generation of photoluminescence after laser ablation on carbon black confined in aqueous phase. In these studies, surface passivation by poly (ethylene glycol) was a driving factor for PL exhibition. Likewise, in carbon nanotubes and carbon dots, luminescence was deeply related to passivation due to the appropriate surface modification which allowed trapping of excitation energy by defect sites in the nanotube structure [29,38,39]. Here, the use of chemically modified MWCNT was an appropriate strategy as after the laser pulse incidence, apparently, both the new nanostructures and the surface modified nanotubes present surface condition that permitted PL emission.

\subsection{Morphology}

To elucidate the physical changes suffered by the nanotubes because of laser pulse a detailed electron microscopy analysis was performed. For comparison purposes, Fig. 5 illustrates the typical morphology of the chemically modified MWCNT before laser irradiation and Fig. 6 portraits micrographs of the nanotubes after irradiation. MWCNT morphology before laser irradiation shows nanotubes with a diameter about $150 \mathrm{~nm}$ and a well-defined surface. Conversely, after laser pulse, nanotubes presented important changes as, for instance, cap opening and partial unzipping; besides, the presence of new abundant nanostructures is also evident, Fig. 6(a). Fig. 6(b) shows the fiber-like morphology of these new nanostructures with a diameter around $20 \mathrm{~nm}$. Additionally, Fig. 6(c) portraits a carbon nanotube exhibiting cap opening and partial unzipping. Furthermore, Fig. 6(d) shows a partially unwinding nanotube evidencing the graphenic structure of nanotube walls. This study revealed the important effect of the laser pulse high energy on the MWCNT structural integrity. Fiberlike nanostructures origin was apparently the product of nanotubes tearing induced by laser incidence, which concurrently produced cap opening and unzipping. Based on these results, we can suggest that nanotube surface modification, combined with the presence of the new carbon nanostructures and chemical 


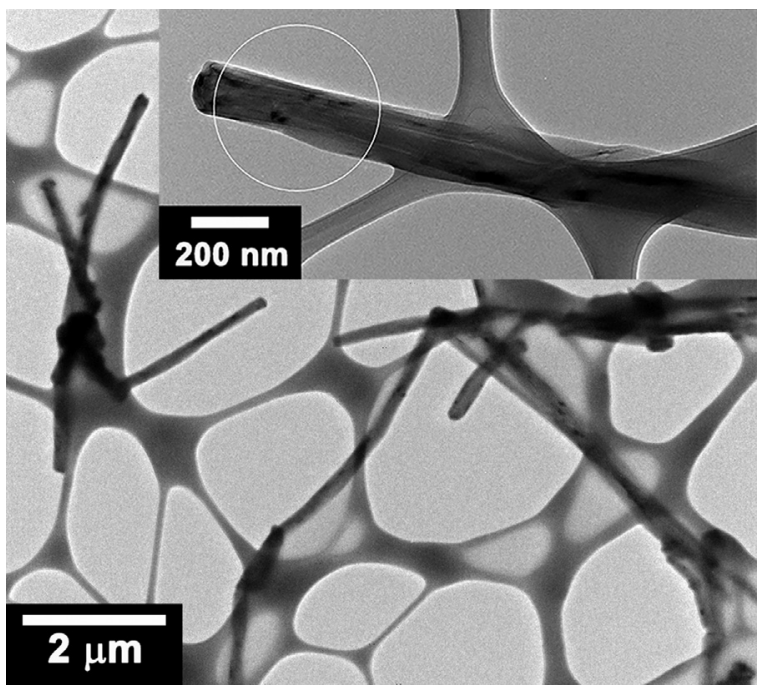

Fig. 5. Micrograph of the multi-walled carbon nanotubes chemically modified before laser irradiation.

modification, were decisive factors in the modification of the optical properties of the irradiated suspension, as discussed above.

To further explore the effect of laser pulses on the nanotubes structure, high-resolution microscopy (HRTEM) characterization was achieved, as shown in Fig. 7. Non-irradiated MWCNT nanostructure (Fig. 7(a)) exhibits the typical thick multi-walled graphitic structure covered by a layer of amorphous carbon. Concerning the irradiated nanotubes, three areas were analyzed, the nanotube surface, the unzipped layer, and the fiber-like structures. From the nanotube surface (Fig. 7(b)), nanocages lower than $10 \mathrm{~nm}$ formed by some few graphitic layers were abundantly observed; however, it is noteworthy that the amorphous phase was also plentiful. The unzipped layers (Fig. 7(c)) showed highly ordered atomic arrangement of carbon atoms, which reflects the graphenic nature of nanotube walls. In the image, only some few layers of graphene are observed. This indicates, indeed, that nanotube unfolding due to laser incidence caused unzipping. Furthermore, the fiber-like arrangement exhibited areas with a partial arrangement; however, the amorphous phase is mostly present (Fig. 7(d)). Literature indicates that over-exposition to laser beam results in the modification of the firstly produced nanostructures. For example, Mikheev et al. [33] reported that photochemical reactions occurring between MWCNT and DMF lead to the formation of new chemical bonds, absent in the initial suspension, besides of impurities and a large number of defects in the nanotubes. Also, Hu et al. [9] showed that when a single carbon black particle is heated by a laser beam, only neighboring graphitic segments within a particle bond grow, which favors the formation of large carbon nanocages. Besides, Tsuji et al. [17] reported that $\mathrm{C}_{60}$ particles, suspended in organic solvents of different polarity, were transformed into disordered $\mathrm{sp}^{2}$ carbon and form graphite-like carbon after the laser irradiation. In the present case, we consider the amorphous nature of fiber-like structures is an evolution of the unzipped layers that lose atomic order due to the over incidence of laser pulses becoming, consequently, amorphous. Selected area electron diffraction (SAED) is included for each structure. SEAD indicates that the non-irradiated nanotube, the unzipped layer, and the nanocages present a crystalline structure, unlike the produced amorphous phase.

Mechanisms leading to nanostructures by laser pulses on solid nanoparticles suspended in liquid media have been proposed. The model reported by Hu et al. [9], for the irradiation of carbon black in an aqueous solution of poly(ethylene glycol), served as an inspiration to explain pulse effect on MWCNT (Fig. 8(a)). According to this model, the high energy of the laser pulse impact produced both the evaporation of the amorphous carbon material contained in the nanotube and the fracture of the graphenic walls (dislocations), as evidenced in Fig. 8(b)) and c). Consequently, the evaporated material as elemental particles (atoms and ions) flowed through those dislocations to the surface of the nanotube. It is


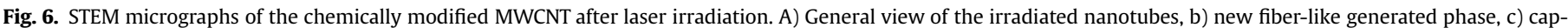
opened and partially unzipped nanotube, and c) unzipped nanotube. 

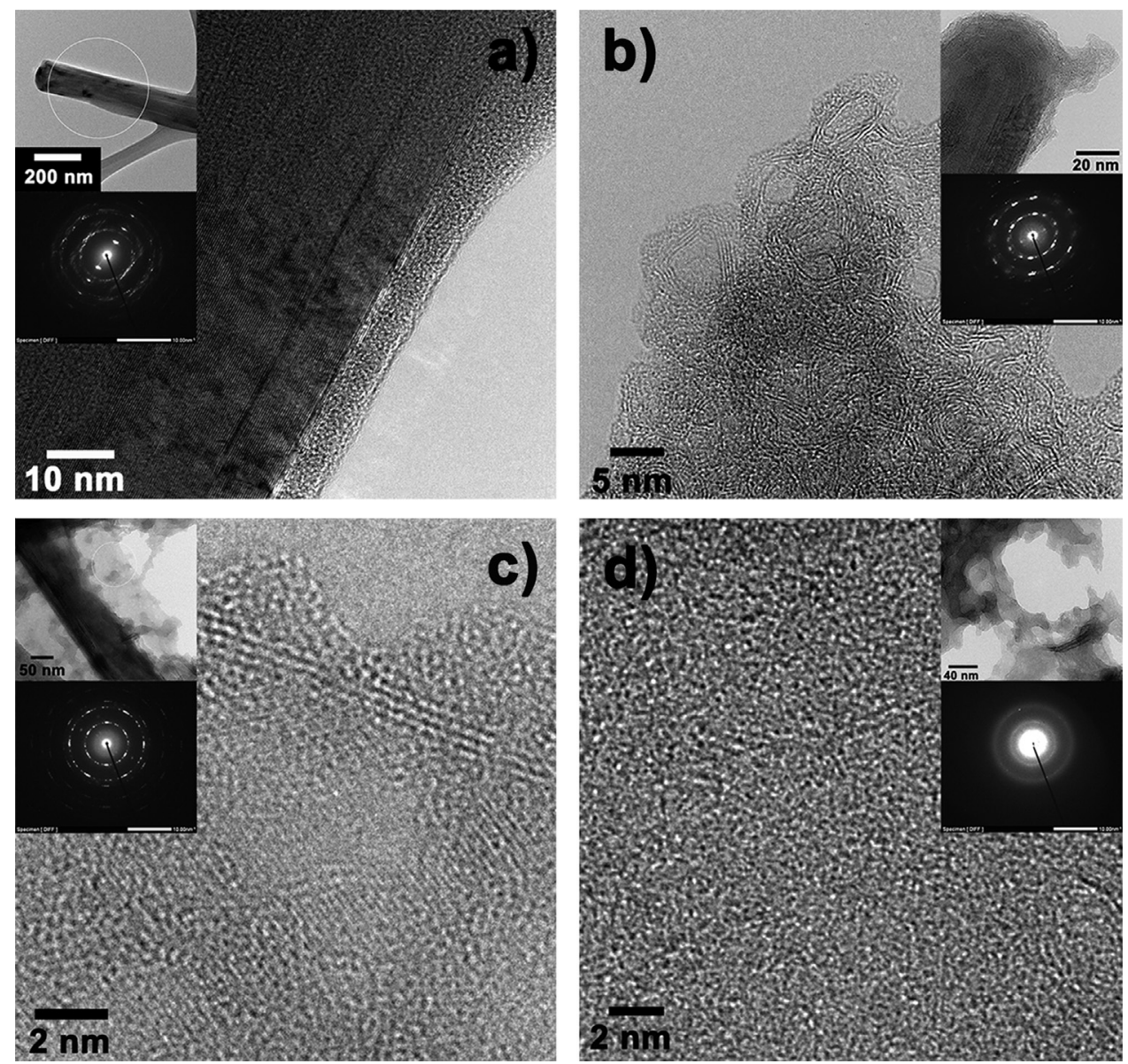

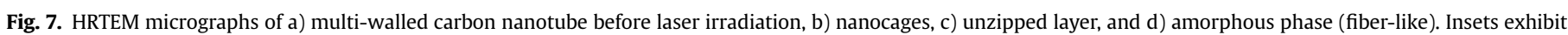
the low magnification areas from where images taken. Selected area electron diffraction (SAED) pattern for each surface is included.

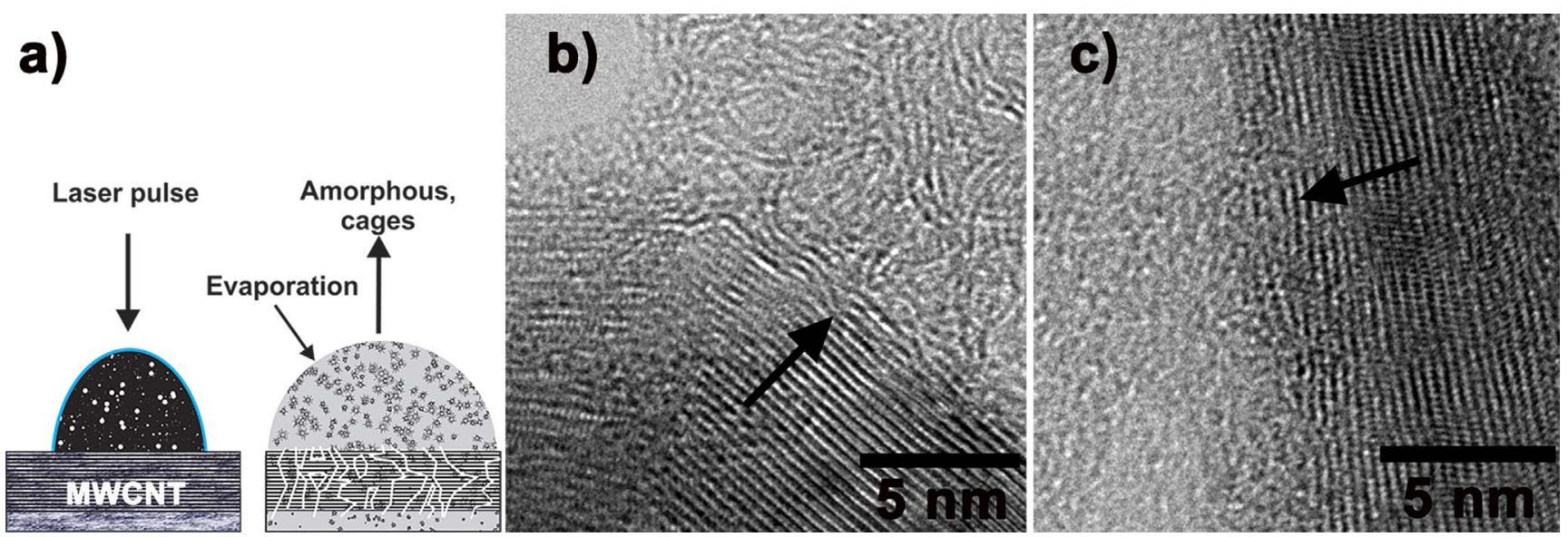

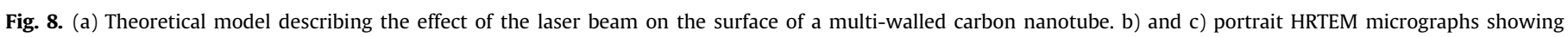
graphenic layer dislocations; the arrows point the direction of flux of the evaporated material product of laser irradiation.

believed that depending on the cooling speed, the re-bonding of the elemental particles permitted the formation of ordered or disordered structures.

\section{Conclusion}

The laser pulse allowed MWCNT microstructure modification, generating graphene-like ordered structures (nanotube unzipping), nanocages, and profuse amorphous carbon, as shown by electron microscopy. The generation of new species of carbon and the surface modification of the carbon nanotubes, produced changes in the optical properties of the suspension, as exhibited by UV-vis spectroscopy and photoluminescence. Laser irradiation, as a tool for the production of carbon nanostructures using as a source of carbon chemically modified MWCNT, is certainly promising. However, more studies are necessary to achieve greater control over the characteristics of the products to establish the conditions to obtain either crystalline or amorphous structures in a controlled way. 


\section{Acknowledgement}

We gratefully acknowledge financial support by "Desarrollo Profesional Docente, para Tipo Superior-Secretaría de Educación Pública, México". We also wish to thank the Laboratorio Nacional de Nanotecnología (CIMAV), as well as Wilber Antunez and Carlos Ornelas for their valuable collaboration during this research.

\section{References}

[1] E. Petryayeva, U.J. Krull, Localized surface plasmon resonance: Nanostructures, bioassays and biosensing-A review, Anal. Chim. Acta 706 (2011) 8-24, http: //dx.doi.org/10.1016/j.aca.2011.08.020.

[2] J.Y. Kim, F.E. Osterloh, ZnO-CdSe nanoparticle clusters as directional photoemitters with tunable wavelength, J. Am. Chem. Soc. 127 (2005) 10152-10153, http://dx.doi.org/10.1021/ja052735f.

[3] S. Naahidi, M. Jafari, F. Edalat, K. Raymond, A. Khademhosseini, P. Chen, Biocompatibility of engineered nanoparticles for drug delivery, J. Control. Release 166 (2013) 182-194, http://dx.doi.org/10.1016/i.jconrel.2012.12.013.

[4] J.D. Mackenzie, E.P. Bescher, Chemical routes in the synthesis of nanomaterials using the sol - gel process, Acc. Chem. Res. 40 (2007) 810-818, http://dx.doi. org/10.1021/ar7000149.

[5] V. Amendola, M. Meneghetti, Laser ablation synthesis in solution and size manipulation of noble metal nanoparticles, Phys. Chem. Chem. Phys. 11 (2009) 3805-3821, http://dx.doi.org/10.1039/b900654k.

[6] D. Reyes-Contreras, M. Camacho-López, M. a Camacho-López, S. CamachoLópez, R.I. Rodríguez-Beltrán, M. Mayorga-Rojas, Influence of the per pulse laser fluence on the optical properties of carbon nanoparticles synthesized by laser ablation of solids in liquids, Opt. Laser Technol. 74 (2015) 48-52, http: //dx.doi.org/10.1016/j.optlastec.2015.05.010.

[7] C.L. Sajti, R. Sattari, B.N. Chichkov, S. Barcikowski, Gram scale synthesis of pure ceramic nanoparticles by laser ablation in liquid, J. Phys. Chem. C. 114 (2010) 2421-2427, http://dx.doi.org/10.1021/jp906960g.

[8] M.F. Becker, J.R. Brock, H. Cai, D.E. Henneke, J.W. Keto, J. Lee, et al., Metal nanoparticles generated by laser ablation, Nanostruct. Mater. 10 (1998) 853-863.

[9] S. Hu, Y. Dong, J. Yang, J. Liu, S. Cao, Simultaneous synthesis of luminescent carbon nanoparticles and carbon nanocages by laser ablation of carbon black suspension and their optical limiting properties, J. Mater. Chem. 22 (2012) 1957-1961, http://dx.doi.org/10.1039/c1jm14510j.

[10] P. Liu, H. Cui, C.X. Wang, G.W. Yang, From nanocrystal synthesis to functional nanostructure fabrication: laser ablation in liquid, Phys. Chem. Chem. Phys. 12 (2010) 3942-3952, http://dx.doi.org/10.1039/b918759f.

[11] E. Akman, O.C. Aktas, B. Genc Oztoprak, M. Gunes, E. Kacar, O. Gundogdu, et al., Fragmentation of the gold nanoparticles using femtosecond Ti:Sapphire laser and their structural evolution, Opt. Laser Technol. 49 (2013) 156-160, http: //dx.doi.org/10.1016/j.optlastec.2013.01.003.

[12] H. Usui, T. Sasaki, N. Koshizaki, Optical transmittance of indium tin oxide nanoparticles prepared by laser-induced fragmentation in water, J. Phys. Chem. B 110 (2006) 12890-12895, http://dx.doi.org/10.1021/jp061866f.

[13] H. Zeng, S. Yang, W. Cai, Reshaping formation and luminescence evolution of $\mathrm{ZnO}$ quantum dots by laser-induced fragmentation in liquid, J. Phys. Chem. C 115 (2011) 5038-5043, http://dx.doi.org/10.1021/jp109010c.

[14] Z. Yan, D.B. Chrisey, Pulsed laser ablation in liquid for micro-/nanostructure generation, J. Photochem. Photobiol. C: Photochem. Rev. 13 (2012) 204-223, http://dx.doi.org/10.1016/j.jphotochemrev.2012.04.004.

[15] H. Zeng, X.-W. Du, S.C. Singh, S.A. Kulinich, S. Yang, I. He, et al., Nanomaterials via laser ablation/irradiation in liquid: a review, Adv. Funct. Mater. 22 (2012) 1333-1353, http://dx.doi.org/10.1002/adfm.201102295.

[16] D. Tan, S. Zhou, J. Qiu, N. Khusro, Preparation of functional nanomaterials with femtosecond laser ablation in solution, J. Photochem. Photobiol. C: Photochem. Rev. 17 (2013) 50-68, http://dx.doi.org/10.1016/j.jphotochemrev.2013.08.002.

[17] M. Tsuii, S. Kuboyama, T. Matsuzaki, T. Tsuii, Formation of hydrogen-capped polyynes by laser ablation of C60 particles suspended in solution, Carbon 41 (2003) 2141-2148, http://dx.doi.org/10.1016/S0008-6223(03)00241-0.

[18] H. Tabata, M. Fujii, S. Hayashi, Laser ablation of diamond particles suspended in ethanol: Effective formation of long polyynes, Carbon 44 (2006) 522-529, http://dx.doi.org/10.1016/i.carbon.2005.08.007.

19] H. Tabata, M. Fujii, S. Hayashi, One-step synthesis of fluorescent carbon nanoparticles by laser irradiation, Eur. Phys. J. 34 (2005) 223-225, http://dx.doi. org/10.1140/epjd/e2005-00147-0.

[20] S.-L. Hu, K.-Y. Niu, J. Sun, J. Yang, N.-Q. Zhao, X.-W. Du, One-step synthesis of fluorescent carbon nanoparticles by laser irradiation, J. Mater. Chem. 19 (2009) 484-488, http://dx.doi.org/10.1039/b812943f.

[21] G.X. Chen, M.H. Hong, T.C. Chong, H.I. Elim, G.H. Ma, W. Ji, Preparation of carbon nanoparticles with strong optical limiting properties by laser ablation in water, J. Appl. Phys. 95 (2004) 1455-1459, http://dx.doi.org/10.1063/ 1.1637933.

[22] D. Tan, Y. Yamada, S. Zhou, Y. Shimotsuma, K. Miura, J. Qiu, Photoinduced luminescent carbon nanostructures with ultra-broadly tailored size ranges, Nanoscale 5 (2013) 12092-12097, http://dx.doi.org/10.1039/c3nr04392d.

[23] S. Hu, Y. Dong, J. Yang, J. Liu, S. Cao, Formation and nonlinear optical properties of carbon nanospindles from laser ablation, CrystEngComm 14 (2012) 4243-4246, http://dx.doi.org/10.1039/c2ce25145k.

[24] A. De Giacomo, A. De Bonis, M. Dell'Aglio, O. De Pascale, R. Gaudiuso, S. Orlando, et al., Laser ablation of graphite in water in a range of pressure from 1 to 146 atm using single and double pulse techniques for the production of carbon nanostructures, J. Phys. Chem. C. 115 (2011) 5123-5130.

[25] D. Tan, S. Zhou, B. Xu, P. Chen, Y. Shimotsuma, K. Miura, et al., Simple synthesis of ultra-small nanodiamonds with tunable size and photoluminescence, Carbon 62 (2013) 374-381, http://dx.doi.org/10.1016/j.carbon.2013.06.019.

[26] S.Z. Mortazavi, P. Parvin, A. Reyhani, S. Mirershadi, R. Sadighi-Bonabi, Generation of various carbon nanostructures in water using IR/UV laser ablation, J. Phys. D: Appl. Phys. 46 (2013) 165303, http://dx.doi.org/10.1088/0022-3727/ 46/16/165303.

[27] S. Hu, F. Tian, P. Bai, S. Cao, J. Sun, J. Yang, Synthesis and luminescence of nanodiamonds from carbon black, Mater. Sci. Eng. B 157 (2009) 11-14, http: //dx.doi.org/10.1016/j.mseb.2008.12.001.

[28] H. Li, X. He, Y. Liu, H. Huang, S. Lian, S.-T. Lee, et al., One-step ultrasonic synthesis of water-soluble carbon nanoparticles with excellent photoluminescent properties, Carbon 49 (2011) 605-609, http://dx.doi.org/10.1016/ j.carbon.2010.10.004.

[29] R. Liu, D. Wu, S. Liu, K. Koynov, W. Knoll, O. Li, An aqueous route to multicolo photoluminescent carbon dots using silica spheres as carriers, Angew. Chem. 121 (2009) 4668-4671, http://dx.doi.org/10.1002/ange.200900652.

[30] S. Fiorito, A. Serafino, F. Andreola, A. Togna, G. Togna, Toxicity and biocompatibility of carbon nanoparticles, I. Nanosci. Nanotechnol. 6 (2006) 591-599, http://dx.doi.org/10.1166/inn.2006.125.

[31] D. Pan, J. Zhang, Z. Li, C. Wu, X. Yan, M. Wu, Observation of pH-, solvent-, spin-, and excitation-dependent blue photoluminescence from carbon nanoparticles, Chem. Commun. 46 (2010) 3681-3683, http://dx.doi.org/10.1039/ c000114.

[32] C. Grabinski, S. Hussain, K. Lafdi, L. Braydich-Stolle, J. Schlager, Effect of particle dimension on biocompatibility of carbon nanomaterials, Carbon 45 (2007) 2828-2835, http://dx.doi.org/10.1016/j.carbon.2007.08.039.

[33] K.G. Mikheev, G.M. Mikheev, V.L. Kuznetsov, T.N. Mogileva, S.I. Moseenkov, M. a Shuvaeva, Laser bleaching of carbon nanotubes suspension in $\mathrm{N}, \mathrm{N}-\mathrm{di}-$ methylformamide, J. Nanomater. Mol. Nanotechnol. 2 (2013) 1-4, http://dx doi.org/10.4172/2324-8777.1000118.

[34] J.E. Moreno-Marcelino, E. Vigueras-Santiago, G. López-Téllez, S. HernándezLópez, Chemical functionalization of carbon nanotubes and its effects on electrial conductivity, J. Nano Res. 28 (2014) 51-61, http://dx.doi.org/10.4028 www.scientific.net/JNanoR.28.51.

[35] N.V. Tarasenko, A.V. Butsen, E. a Nevar, N.A. Savastenko, Synthesis of nanosized particles during laser ablation of gold in water, Appl. Surf. Sci. 252 (2006) 4439-4444, http://dx.doi.org/10.1016/j.apsusc.2005.07.150.

[36] P.-H. Chung, E. Perevedentseva, C.-L. Cheng, The particle size-dependent photoluminescence of nanodiamonds, Surf. Sci. 601 (2007) 3866-3870, http: //dx.doi.org/10.1016/j.susc.2007.04.150.

[37] Y. Sun, B. Zhou, Y. Lin, W. Wang, K.A.S. Fernando, P. Pathak, et al., QuantumSized Carbon Dots for Bright and Colorful Photoluminescence, J. Am. Chem. Soc. 128 (2006) 7756-7757, http://dx.doi.org/10.1021/ja062677d.

[38] Y. Lin, B. Zhou, R.B. Martin, K.B. Henbest, B.A. Harruff, J.E. Riggs, et al., Visible luminescence of carbon nanotubes and dependence on functionalization, I. Phys. Chem. B 109 (2005) 14779-14782, http://dx.doi.org/10.1021/jp053073j.

[39] J.E. Riggs, Z. Guo, D.L. Carroll, Y.-P. Sun, Strong luminescence of solubilized carbon nanotubes, J. Am. Chem. Soc. 122 (2000) 5879-5880. 